\title{
LAS TIC EN LA EDUCACIÓN SUPERIOR COMO VÍA DE FORMACIÓN Y DESARROLLO COMPETENCIAL EN LA SOCIEDAD DEL CONOCIMIENTO
}

\section{ICT IN HIGHER EDUCATION AS A MEANS OF TRAINING AND SKILLS DEVELOPMENT IN THE KNOWLEDGE SOCIETY}

Ángela María Molina Ramírez (anyara@correo.ugr.es). Universidad de Granada

Fecha de recepción: 28 de mayo de 2012
Fecha de aceptación: 11 de junio de 2012
Localizador: http:www.ugr.es/local/miguelgr/ReiDoCrea-Vol.1-Art.15-Molina.pdf

"Si le das pescado a un hombre hambriento,

lo nutres durante una jornada.

Si le enseñas a pescar,

lo nutrirás durante toda su vida"

Lao-Tsé

\section{RESUMEN}

La sociedad del conocimiento, nos pone a prueba en relación a las destrezas, conocimientos y habilidades que adquirimos. El ámbito educativo ha de avanzar al compás de esta sociedad desde la primera de las etapas hasta el final de ellas, valiéndose de las TIC. A través del presente trabajo se pretende analizar la interacción comunicativa establecida entre docentes y discentes a través de los espacios virtuales en la Educación Superior, profundizando en sus posibilidades y destacando la necesidad de flexibilizar la educación. A la vez, se muestran una serie de experiencias renovadoras y unas vías de continuidad en el futuro.

Palabras clave: tutor virtual, espacio virtual, aprendizaje virtual, TIC, Educación Superior.

\section{ABSTRACT}

The knowledge society, tests us in relation to the skills, knowledge and abilities we acquire. The education sector has to move in time with this society from the first of the stages to the last, using ICT. The aim of this article is to analyze the communicative interaction established between teachers and students through virtual spaces in higher education, exploring its capabilities and emphasizing the need for flexibility in education. A series of renovating experiences and possibilities for future continuity are also shown.

Key words: virtual tutor, virtual space, e-learning, ICT, Higher Education. 


\section{INTRODUCCIÓN}

En la actualidad, con el auge de las Tecnologías de la Información y la Comunicación (TIC), y especialmente los servicios y posibilidades que ofrece Internet, la formación en red proporciona una enseñanza personalizada y un seguimiento continuo de los progresos del alumnado, optimizando el proceso de aprendizaje y eliminando barreras espacio-temporales. Este entorno nos va a facilitar la comunicación e interacción entre los distintos agentes (profesorado-alumnado-personal administrativo-institución), configurando así diferentes espacios comunicativos: para la tutoría, para la comunicación social, de soporte en las diferentes situaciones didácticas, así como en las organizativas, entre otras.

Pero trabajar con estos ambientes requiere un cambio de mentalidad en la forma de concebir la educación en general, y el proceso de enseñanza-aprendizaje en particular, debido a que son necesarias profundas modificaciones en su consideración y puesta en práctica, empezando por el cambio de rol tanto de profesorado, como del alumnado, y de las propias instituciones educativas. Se hace necesario formar tanto a tutores/as como alumnos/as de Educación Superior en e-learning, en la medida en que la influencia del profesorado puede ser determinante para el acercamiento del alumnado hacia el conocimiento y la interacción comunicativa, hasta tal punto que puede convertirse en la génesis de los procesos motivacionales hacia el aprendizaje. Esto deja patente que la competencia emocional del docente no incide únicamente en paliar el malestar afectivo del profesorado, asimismo adquiere un gran valor por su posible contribución sobre los alumnos y su aprendizaje.

\section{LAS TIC, RESORTE Y TRAMPOLÍN PARA LA COMUNICACIÓN EN LA EDUCACIÓN SUPERIOR}

\section{Aproximación conceptual: marco de referencia.}

Como se ha apuntado en la introducción, la nueva sociedad del conocimiento y la tecnología precisa que la educación y este nuevo avance en el mundo digital lleven caminos paralelos para que, desde la primera etapa educativa (Educación Infantil) hasta la Educación Superior tenga cada vez más cabida el mundo de las nuevas tecnologías y nos facilite el tener contacto con nuevas experiencias de aprendizaje y logre un amplio desarrollo de las capacidades que desde el informe Delors se nos solicita: aprender a conocer, aprender a hacer, aprender a vivir juntos, y aprender a ser.

Es necesario aunar las ventajas de las TIC a la Educación Superior y a la educación en su totalidad, y, como expone Cristóbal Cobo Roman, investigador de Oxford Internet Institute, en una entrevista concedida para "Cuadernos de Pedagogía" , huir de la dura realidad que en ocasiones acontece, y es que "cambian las tecnologías, pero se replican los patrones y los errores del pasado", por lo que a veces, se actúa desde dos universos paralelos.

\begin{tabular}{|l|l|}
\hline MUNDO DIGITAL & MUNDO EDUCATIVO \\
\hline $\begin{array}{l}\text { Crecimiento tecnológico continuo y sin } \\
\text { precedentes que fomenta la }\end{array}$ & $\begin{array}{l}\text { Estancamiento institucional; cambio } \\
\text { fragmentado e insostenible que dificulta }\end{array}$ \\
comunicación entre las personas, la & el aprendizaje y el desarrollo profesional. \\
autoría y la adquisición de conocimiento. & $\begin{array}{l}\text { Inversiones insuficientes, mucho menos } \\
\text { para la investigación, el desarrollo y la } \\
\text { Inversiones millonarias para desarrollar } \\
\text { nuevos productos. }\end{array}$ \\
\hline
\end{tabular}




\begin{tabular}{|l|l|}
\hline Expresión libre. & $\begin{array}{l}\text { Expresión controlada académicamente. } \\
\text { Respuesta constante y múltiple. }\end{array}$ \\
$\begin{array}{l}\text { Expresión multimodal (aural, oral, textual, } \\
\text { imagen, música). }\end{array}$ & $\begin{array}{l}\text { Expresión monomodal (sobre todo } \\
\text { textual). } \\
\text { Identidad móvil. }\end{array}$ \\
Entornos cambiantes. & $\begin{array}{l}\text { Identidad fija. } \\
\text { Entornos constantes. } \\
\text { Multiárea. }\end{array}$ \\
Rapidez. & Tareas consecutivas. \\
\hline
\end{tabular}

No se trata, pues, de mantener estos mundos separados, sino involucrar las TIC en la educación, en este caso en la Educación Superior. De esta idea surgen los entornos virtuales como alternativa metodológica a la educación tradicional.

Entendemos por un entorno virtual de aprendizaje aquel espacio o comunidad organizados con el propósito de lograr el aprendizaje y que para que éste tenga lugar requiere ciertos componentes (Salinas, 2004c): una función pedagógica, la tecnología apropiada a la misma y los aspectos organizativos.

En la educación superior, estos sistemas presentan grandes oportunidades tanto para los docentes como para los estudiantes, en términos de accesibilidad, de flexibilidad y, en algunos casos, de costos. Impactan, por tanto, en tres de los aspectos críticos de la educación superior actual: en la necesidad de proporcionar acceso a una cantidad cada vez mayor a la educación postsecundaria, en la necesidad de modalidades cada vez más flexibles en términos de lugar, espacio, ritmo, itinerarios, etc. y en la importancia que va tomando la financiación y, en consecuencia, los costos de la educación superior.

\section{La educación flexible como necesidad y punto de partida.}

Para Latona (1996), las universidades deben tomar el enfoque de la formación flexible, ya que se han de adaptar a las tendencias de acceder al aprendizaje fuera del puesto de trabajo, proveer las necesidades de los estudiantes a tiempo parcial y responder a las necesidades de formación continua, que constituyen desafíos para las instituciones de educación superior en esta sociedad de la información.

Como concepto y como práctica, el aprendizaje flexible en la Educación Superior arrastra cualidades o experiencias de sus tres bases (Salinas, 1999): de la educación a distancia, de la educación en el campus, y de las tecnologías de la información y la comunicación. En este ámbito, la formación flexible, sirve tanto para aquellos alumnos que siguen la enseñanza 'presencial', como para aquéllos que siguen la enseñanza a distancia o por cualquiera de las fórmulas mixtas.

¿Cómo llevar a cabo la flexibilización de la educación? En primer lugar, en el momento de organizar estos entornos virtuales de enseñanza-aprendizaje pueden diferenciarse distintos componentes que se interrelacionan y condicionan y se han de tener en cuenta. La preocupación por los mismos varía desde las herramientas que facilitan el entorno (De Benito, 2000), o el rol del profesor (Gisbert, 1997; Salinas, 1999), a las estrategias didácticas a desplegar en dicho entorno (Pérez i Garcías, 2002, 2004), la evaluación de los entornos (Salinas, 2004), la perspectiva organizativa (Duart y Lupiañez, 2005), los modelos (Sangrà y Duart, 1999; Henríquez, 2001), etc... 
Las modalidades de formación apoyadas en las TIC llevan a nuevas concepciones del proceso de enseñanza-aprendizaje que acentúan la implicación activa del alumnado en el proceso de construcción de conocimiento; la atención a las destrezas emocionales e intelectuales a distintos niveles; la preparación de los jóvenes para asumir responsabilidades en un mundo en rápido y constante cambio, la flexibilidad para entrar en un mundo laboral que demandará formación a lo largo de toda la vida y las competencias necesarias para este proceso de aprendizaje continuo (Salinas, 1997).

Algunos de estos cambios se reflejan en la siguiente tabla:

\begin{tabular}{|l|l|l|}
\hline $\begin{array}{l}\text { FUNCIONES DE LA } \\
\text { INSTITUCIÓN } \\
\text { UNIVERSITARIA }\end{array}$ & $\begin{array}{l}\text { GESTIÓN DEL } \\
\text { CONOCIMIENTO }\end{array}$ & UTILIZACIÓN DE LAS TIC \\
\hline Investigación & Creación, generación & $\begin{array}{l}\text { Laboratorios virtuales } \\
\text { Recursos de investigación } \\
\text { Grupos distribuidos } \\
\text { Creación compartida de recursos }\end{array}$ \\
\hline Docencia & Transmisión & $\begin{array}{l}\text { Campus virtuales } \\
\text { E-learning, e-campus }\end{array}$ \\
\hline Preservación & Codificación, archivo & $\begin{array}{l}\text { Ribliotecas virtuales } \\
\text { Redes de bibliotecas } \\
\text { Informatizadas }\end{array}$ \\
\hline $\begin{array}{l}\text { Proyección, } \\
\text { transferencia }\end{array}$ & Aplicación & $\begin{array}{l}\text { Revistas y publicaciones } \\
\text { electrónicas, e-books }\end{array}$ \\
\hline Extensión, publicación & Divulgación & $\begin{array}{l}\text { Transferencia de conocimiento en } \\
\text { red } \\
\text { Innovación } \\
\text { Redes de aprendizaje }\end{array}$ \\
\hline
\end{tabular}

En relación al proceso de enseñanza-aprendizaje, Moore (1989), Bates (1991), Berge y Collins (1996) o Gilbert y Moore (1998) hablan de los varios tipos esenciales de interacción que Salinas (2004) recoge como tres dimensiones de los entornos virtuales:

Interactividad del material (Interacción Alumno-Contenido).

Apoyo y comunicación (Interacción Profesor-Alumno).

Reconstrucción del grupo de aprendizaje que se concreta en la organización de la comunidad de aprendizaje (Interacción Alumno-Alumno). 
En definitiva, reflexionar sobre cambios metodológicos en los entornos virtuales implica cambios en los otros elementos. De ahí que parezca que la llegada de las TIC genera procesos de innovación como señalábamos antes (o que implique cambios más profundos que los de la simple introducción de un elementos nuevo en el proceso).

Ésta tarea no es fácil, y los retos que para la organización del proceso de enseñanzaaprendizaje dependerán en gran medida del escenario de aprendizaje, (el hogar, el puesto de trabajo o el centro de recursos de aprendizaje, etc.).

En cualquiera de las circunstancias, en la gestión de los entornos virtuales de formación habrá de tener en consideración a todos y cada uno de los componentes del modelo de educación flexible y las distintas relaciones que pueden establecerse entre los mismos.

\section{Aterrizaje: posibilidades que las TIC ofrecen a través de la comunicación.}

Actualmente, las universidades han de plantear nuevos modelos en el contexto en el aula convencional, y desde una perspectiva flexible atender a las personas que necesitan formación a lo largo de la vida, explorando cómo la tecnología puede contribuir a implicar y apoyar a los alumnos en contextos, culturas y programas múltiples.

¿Qué implica todo esto? Supone nuevos entornos de aprendizaje y de enseñanza, lo que requiere nuevos enfoques para entenderlos, diseñarlos y gestionarlos. Al respecto, los autores Mason y Kaye (1990), han señalado que la aplicación de la comunicación mediada por ordenador ha cambiado la naturaleza y estructura de las instituciones de educación a distancia de diferentes formas, indicando tres implicaciones:

Desaparición de las distinciones conceptuales entre educación a distancia y educación presencial.

Cambio de los roles tradicionales del profesorado, tutores adjuntos y staff administrativo y de apoyo.

Oportunidad de crear una red de estudiantes, un 'espacio' para el pensamiento colectivo.

¿Cómo se lleva a la práctica? Gestionar un entorno de formación supone un conjunto de decisiones en forma de juego de equilibrio entre el modelo pedagógico, las posibilidades de la tecnología y el marco organizativo e institucional. Una vez consensuada y tomada la decisión, lo que las universidades tienen que hacer es construir un nuevo entorno de aprendizaje que intencional y sistemáticamente (Hanna, 2000):

Conecte a los alumnos unos con otros y con los tutores y profesores.

Conecte este aprendizaje con la experiencia.

Saque ventaja de la abundancia de información y accesible mediante las nuevas tecnologías.

Así pues, ¿qué perfil tienen el estudiante y el tutor en los espacios virtuales?

Perfil del estudiante en los espacios virtuales 
Como establece Monereo (2005), a nuestros jóvenes nadie les ha enseñado a buscar y seleccionar información de manera crítica, parafraseando y filtrando lo que leen, y recelando de determinadas fuentes, medios o autores, y es ahí donde hemos de incidir. Gracias a los espacios virtuales, el protagonista del proceso es el alumno, el cual puede preguntar al profesor diez veces más en e-learning que en la modalidad presencial, y el autoaprendizaje adquiere un papel relevante.

Junto al enorme potencial del uso de las tecnologías, hoy sabemos que todo aprendizaje es el resultado de un proceso interactivo (otras personas, contexto propio, otros contextos) (Moreno, 2003) y que existe una correspondencia formal entre los modelos cognitivos en la memoria y la estructura de la web.

Las nuevas tecnologías y las demandas de nuevas competencias han ampliado el espectro de los usuarios respecto al alumnado tradicional que ha finalizado la enseñanza secundaria obligatoria. Cada vez más aumenta la variabilidad del alumno medio, el cual cada vez es de mayor edad, y a menudo, compagina los estudios con el trabajo, capaz de absorber contenidos educativos con facilidad en los distintos formatos, es una persona que difícilmente consigue adaptarse al horario rígido de las clases y por tanto precisa de la flexibilidad de la educación.

De este modo, nos vemos obligados a considerar las diferencias de los alumnos desde el punto de vista de la motivación, de la percepción, desde los estilos cognitivos. Además, es preciso apuntar a un grupo de habilidades y actitudes básicas personales para aprender en la distancia: autodisciplina, la capacidad de trabajar solo, la gestión del tiempo, cierto grado de independencia en el aprendizaje; capacidad de realizar una programación personal, compaginando vida personal, profesional y estudios.

Por tanto que el e-learning es un modelo de aprendizaje en el que el estudiante tiene que desarrollar habilidades tan importantes para su vida futura en esta sociedad, y donde se considera el medio óptimo para el desarrollo de competencias (conocimientos, habilidades y actitudes).

\section{Perfil del tutor en los espacios virtuales}

El mejor tutor es el que a medida que enseña se va haciendo más prescindible, al tiempo que el estudiante va adquiriendo cada vez más autonomía (García Nieto, 2004).

Se pretende formar estudiantes autónomos, que autodirijan convenientemente su proceso de aprendizaje permanente. En este caso, el tutor virtual debe conocer y adherir las demandas que plantea la sociedad de la información reflejada de manera sintética en el perfil del estudiante ya descrito.

Trabajar en línea crea una amplia serie de sensaciones en los participantes y los tutores on-line. Además de la función didáctica tradicional, el docente está llamado ahora a desempeñar otras funciones de tipo social, de gestión y tecnológicas, las que son funciones del equipo e-learning (Ardizzone, 2004), es un mediador entre la información y el conocimiento (Tribó, 2005).

Los tutores necesitan formación para evaluar y corregir electrónicamente (Salmon, 2004), especialmente en modalidades de evaluación definidas desde el diseño curricular de la experiencia formativa, las pruebas de desempeño, las evidencias de conocimiento, etc. (Inacap, 2007; Irigoin, 2002).

El buen tutor virtual es experimentado y entusiasta, implicado en la formación, transmite entusiasmo; flexible, pero organizado; tolerante, buen comunicador y mejor "oyente", conocedor de la tecnología. 
La experiencia apunta que los estudiantes prefieren que las respuestas sean personalizadas y dirigidas a su trabajo específico. Todas las interacciones y respuestas deben servir a un propósito. El tutor virtual cumple dichas expectativas y necesidades.

\section{EXPERIENCIAS RENOVADORAS}

A partir del curso 2006-2007 se plantea en la Universidad de Sevilla la conversión y uso de materias a través de la plataforma WebCT. Blackboard Learning SystemWebCT es una plataforma informática de teleformación (e-learning) que permite construir y administrar cursos en línea e impartir formación, ofertada al profesorado como complemento a la docencia presencial.

WebCT es un software que permite a las instituciones educativas crear y alojar cursos en internet que pueden servir como cursos en línea completos o como complemento a los cursos magistrales tradicionales (Añel, 2008; León, 2007), donde su principal ventaja es la sincronización que se realiza con los sistemas de gestión corporativos académicos de la universidad.

En el curso 2007-2008 ya se empezaron a ver los resultados y fueron tan sorprendentes que la Universidad de Sevilla ha decidido abordar la ampliación del abanico de proyectos y entornos colaborativos tales como el OpenCourseWare (OCW), iniciativa surgida en el Instituto Tecnológico de Massachussets en 2001. En este sentido, tienen publicado el material didáctico de las materias Psicología de los Grupos y de las Organizaciones, impartida en las titulaciones de Psicología, Pedagogía y Psicopedagogía.

Mi propia experiencia como alumna de Educación Infantil y de Psicopedagogía me ha hecho reflexionar sobre la importancia del aprendizaje y la formación a través de plataformas y entornos virtuales. La Universidad de Granada ofrece al alumnado varias vías de comunicación, donde se encuentra el Tablón de Docencia como espacio de comunicación entre profesorado y alumnado y lugar donde se pueden depositar materiales y recursos de las materias.

Además, SWAD, MOODLE, CEVUG, Ágora de Psicología son otra serie de plataformas que se ofrecen al alumnado para el desempeño del seguimiento didáctico, donde se ponen al servicio de todos y todas diversas vías de comunicación sincrónica y asincrónica, tales como chat, videoconferencia, correo electrónico, foros, calendario, tablón de anuncios, carpetas compartidas, etc.

Actualmente, Gmail ofrece una maravillosa oportunidad de trabajar en línea. De forma personal, a través de la herramienta Docs curso la asignatura Psicología de los Grupos de la titulación Psicopedagogía, donde el profesor y yo nos comunicamos en función de las tareas que voy realizando.

Opciones como las comentadas ofrecen amplia flexibilidad a las personas que por diversas circunstancias no pueden acudir de forma presencial a los cursos que se soliciten. Además, es un avance y una nueva forma de comunicación atractiva y productiva que yo misma he podido comprobar a lo largo de mi experiencia como docente y como maestra.

\section{TRES CAMINOS HACIA EL FUTURO}

Un buen sistema educativo integra el método de enseñanza y la evaluación con las actividades descritas en los objetivos, de modo que todos los elementos del sistema funcionan conjuntamente para favorecer el aprendizaje (Lebrun, 2007). ¿Cómo 
obtener esta integración? Existen tres estrategias que responden a la demanda tecnológica (Underwood y Dillon, 2011).

\section{Minimizar el uso de la tecnología.}

Los estudiantes que pueden acceder a la tecnología fuera del aula tienen una ventaja, con lo que se crea una diferencia con respecto a los que no tienen los medios económicos o culturales que les permite disponer de acceso a estos recursos.

\section{Hacer uso de la tecnología para complementar la práctica actual.}

Aceptar la tecnología en aquellos lugares donde encaja, según los métodos y las estructuras actuales. Esta opción define la tecnología como un recurso útil y que posee el valor de catalizador del cambio. En este caso, nos encontramos con que hay innovaciones mejor integradas en el aula, como las pizarras digitales, mientras que otras más utilizadas, como los teléfonos 3G, apenas tienen un impacto en este sistema (Underwood y Dillon, 2011).

\section{Unificar y evolucionar.}

Los profesores y los centros necesitan convertirse en instituciones educativas abiertas que "cambian radicalmente su perspectiva acerca del conocimiento, la evaluación y la relación entre estudiante, maestro e información” (Hernández y Goodison, 2004).

Cualquier intento de integrar la tecnología requiere cierta alteración del actual sistema educativo. Ignoramos un hecho fundamental: el mundo de las tecnologías digitales ha transformado al estudiante; nosotros, como maestros, ¿nos transformaremos con ellos?

\section{CONCLUSIONES}

En un amplio número de universidades europeas se ha observado que la metodología está ya más enfocada en el aprendizaje centrado en el alumnado, en el fomento de competencias y en el acompañamiento tutorial, mientras que en España el peso de la docencia pivota todavía mayoritariamente sobre la clase magistral, por lo que debemos trabajar para modificar esta situación.

Por tanto, se trata de crear un nuevo entorno de aprendizaje que fomente la comunicación entre docente y discente, que conecte el aprendizaje con la experiencia, que potencie el trabajo en grupo y aproveche la cantidad de información y conocimiento que se ofrece, dando lugar a comunidades de aprendizaje universitarias.

\section{REFERENCIAS BIBLIOGRÁFICAS}

Adell, J. y Sales, A. (2000). Enseñanza online: elementos para la definición del rol del profesor, en AAVV. Las nuevas tecnologías para la mejora educativa. Kronos, Sevilla, p. 351-372.

Añel, M. E. (2008). Formación on-line en la Universidad. Pixel-Bit. Revista de Medios y Educación, n. ํㅜㅇ, p. 155-163.

Bernal Guerrero, A. y Cárdenas Gutiérrez, A. R. (2009). Influencia de la competencia emocional docente en la formación de procesos motivacionales e identitarios en estudiantes de Educación Secundaria. Una aproximación desde la memoria autobiográfica del alumnado. Revista de Investigación Educativa, n. ำ 1, p. 203-222.

Cabrero, J. (2006). Bases pedagógicas del e-learning. Revista de Universidad y Sociedad del Conocimiento (RUSC), vol. 3, n. $\stackrel{\circ}{1}$. 
García González, A. J., Troyano Rodríguez, Y., Curral, L., Chambel, M. a J. (2010). Aplicación de herramientas de comunicación de la plataforma WEBCT en la tutorización de estudiantes universitarios dentro del Espacio Europeo de Educación Superior. Pixel-Bit. Revista de Medios y Educación, n. ํ37, p. 159-170.

García Ramírez, J. M. (2011). Una reconsideración de la excelencia visible en la educación superior: la escuela empática. En Andalucía Educa, n. ํ 66, p. 77.

García Ramírez, J. M. (2012). Las Tecnologías de la Información y la Comunicación, TIC, en la educación universitaria. En Andalucía Educa, n. ำ 76, p. 84-85.

Gisbert, M. (2002). El nuevo rol del profesor en entornos tecnológicos. En Acción Pedagógica, vol. 11, n. ำ 1, p. 48-59.

Junta de Andalucía (2011). TIC e innovación. Cuadernos de Pedagogía, n. o 418 .

Llorente Cejudo, M. a C. (2005). La tutoría virtual: técnicas, herramientas y estrategias. Universidad de Sevilla.

Marín Sánchez, M. (2003). La interacción comunicativa en los grupos. En Marín Sánchez, M., Garrido Torres, M. A. (2003), El grupo desde la perspectiva psicosocial. Conceptos básicos. Madrid: Pirámide.

Salinas, J. (2005). La gestión de los entornos virtuales de formación. En Seminario Internacional: La Calidad de la Formación en Red en el Espacio Europeo de Educación Superior. 